\title{
Asteroseismology of the exoplanet-host F-type star 94 Ceti: Impact of atomic diffusion on the stellar parameters
}

\author{
M. Deal ${ }^{1,3}$, M. E. Escobar ${ }^{2,4}$, S. Vauclair ${ }^{2,3}$, G. Vauclair ${ }^{2,3}$, A. Hui-Bon-Hoa ${ }^{2,3}$, and O. Richard ${ }^{1}$ \\ ${ }^{1}$ Laboratoire Univers et Particules de Montpellier (LUPM), UMR 5299, Université de Montpellier, CNRS, \\ Place Eugène Bataillon, 34095 Montpellier Cedex 5 France \\ e-mail: morgan.deal@umontpellier.fr \\ 2 Université de Toulouse, UPS-OMP, IRAP, 31400 Toulouse, France \\ ${ }^{3}$ CNRS, IRAP, 14 avenue Edouard Belin, 31400 Toulouse, France \\ ${ }^{4}$ Programa Explora, CONICYT, Ministerio de Educación, Moneda 1375, Santiago, Chile
}

Received 1 October 2016 / Accepted 8 February 2017

\begin{abstract}
Context. A precision of the order of one percent is needed on the parameters of exoplanet-hosts stars to correctly characterise the planets themselves. This can be achieved by asteroseismology. It is important in this context to test the influence of introducing atomic diffusion with radiative accelerations in the models upon the derived parameters. In this paper, we begin this study with the case of the star 94 Ceti A.

Aims. We aim to perform a complete asteroseismic analysis of the exoplanet-host F-type star 94 Ceti A, from the first radial-velocity observations with the High Accuracy Radial velocity Planet Searcher (HARPS) up to the final computed best models. We also aim to test the influence of atomic diffusion, including radiative accelerations, upon the computed frequencies and on the determined stellar parameters. 94 Ceti $\mathrm{A}$ is hot enough to suffer these effects. We also aim to test the effect of including a complete atmosphere in the stellar models.

Methods. The radial velocity observations were performed with HARPS in 2007 . The low degree modes were derived and identified using classical methods and compared with the results obtained from stellar models computed with the Toulouse Geneva Evolution Code (TGEC).

Results. We obtained precise parameters for the star 94 Ceti A. We showed that including atomic diffusion with radiative accelerations can modify the age by a few percent, whereas adding a complete atmosphere does not change the results by more than one percent. Conclusions. Atomic diffusion including radiative accelerations should be taken into account in all the computations of stellar models with masses larger than $1.3 M_{\odot}$ in order to determine accurate parameters for observed stars.
\end{abstract}

Key words. asteroseismology - planetary systems - diffusion - instabilities - stars: evolution

\section{Introduction}

Asteroseismic studies of $\mathrm{G}$ and F-type stars were strongly boosted by space observations, especially with the Kepler satellite. Big data have been obtained, leading to global studies referred to as "ensemble asteroseismology". However, it is still important to study particular stars for deeper insights, including detailed internal physical structure. Radial velocity observations of the exoplanet-host star 94 Ceti obtained with the High Accuracy Radial velocity Planet Searcher (HARPS) allowed derivation of seismic frequencies precise enough to test the importance of new stellar physics on the derived parameters. This star is on the main sequence, hot enough to suffer the effects of atomic diffusion including radiative accelerations on heavy elements in an important way. This leads to element accumulation in specific internal layers and to extra mixing (Deal et al. 2016). We thought it would be interesting to test, at least in a preliminary way, this new physics on an F-type star through asteroseismology. We also wanted to study the impact of introducing a complete atmosphere instead of the usual grey limit.

94 Ceti is a binary system. An exoplanet has been found

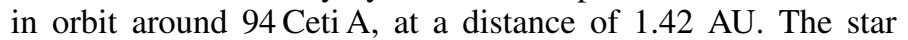
94 Ceti A is classified as an F8V star, with a visual magnitude of $V=5.08$. Its parallax, $\pi=44.29 \pm 0.28$ mas (HIPPARCos catalogue, van Leeuwen 2007), puts it at a distance of $d=$ 22.38 pc. Its companion is an $\mathrm{M}$ dwarf that is 6.4 mag fainter at a distance of approximately $100 \mathrm{AU}$ (Hale 1994).

In the present paper, we first obtain the parameters of the star 94 Ceti A (HD 19994, HR 962, HIP 14954, GJ 128) by using classical standard evolutionary models and fitting these models with the observations gathered with the spectrograph HARPS. Then we obtain parameters with more sophisticated models including detailed radiative accelerations, as computed in the Toulouse Geneva Evolution Code (TGEC), and discuss them. Finally, we add complete atmospheres to characterise the impact of more sophisticated treatments of the stellar outer layers.

\section{The observational data}

\subsection{Spectroscopic observations}

Several spectroscopic studies have been performed for 94 Ceti A. Some of them suggest that it has a nearly solar metallicity, such as Edvardsson et al. (1993) who, in their study of the chemical evolution of the galactic disk, gave it a metallicity of 0.09. Eight years later, Smith et al. (2001) gave a similar metallic value for this star in their work exclusively focused on 94 Ceti A and its abundance distribution. However most studies mainly focused 
Table 1. Summary of previous spectroscopic studies of 94 Ceti.

\begin{tabular}{cccl}
\hline \hline$[\mathrm{Fe} / \mathrm{H}]$ & $T_{\mathrm{eff}}(\mathrm{K})$ & $\log g$ & References \\
\hline $0.09 \pm 0.10$ & $6104 \pm 100$ & $4.10 \pm 0.20$ & Edvardsson et al. (1993) \\
$0.09 \pm 0.05$ & $6030 \pm 20$ & $3.95 \pm 0.05$ & Smith et al. (2001) \\
$0.25 \pm 0.08$ & $6217 \pm 67$ & $4.29 \pm 0.08$ & Santos et al. (2004) (CORALIE) \\
$0.32 \pm 0.07$ & $6290 \pm 58$ & $4.31 \pm 0.13$ & Santos et al. (2004) (FEROS) \\
$0.19 \pm 0.05$ & $6121 \pm 33$ & $4.06 \pm 0.05$ & Santos et al. (2004) (UVES) \\
$0.21 \pm 0.08$ & $6132 \pm 67$ & $4.11 \pm 0.23$ & Santos et al. (2004) (UES) \\
$0.19 \pm 0.03$ & $6188 \pm 44$ & $4.24 \pm 0.06$ & Valenti \& Fischer (2005) \\
$0.19 \pm 0.03$ & $6140 \pm 31$ & $4.35 \pm 0.09$ & Maldonado et al. (2012) \\
\hline
\end{tabular}

Table 2. Summary of HD 19994b properties (Mayor et al. 2004).

\begin{tabular}{lc}
\hline \hline HD $19994 \mathrm{~b}$ & \\
\hline$M \sin i$ & $1.68 M_{\mathrm{J}}$ \\
Orbital period & $535.7 \pm 3.1$ days \\
Semi-major axis & $1.42 \mathrm{AU}$ \\
Eccentricity & $0.30 \pm 0.04$ \\
$\omega$ & $211 \pm 6$ \\
\hline
\end{tabular}

on the exoplanet host-star status of 94 Ceti and led to a stellar overmetallicity. This is the case for Santos et al. (2001, 2003, 2004), who present 94 Ceti amongst their general studies of statistical properties, metal-rich nature, and spectroscopic $[\mathrm{Fe} / \mathrm{H}]$ for exoplanet-host stars. In our study, we use the metallicity values of their 2004 work, which presents a summary and a revision of their previous results and provides separate values for $[\mathrm{Fe} / \mathrm{H}]$ derived from the high-resolution spectra obtained with four different instruments: CORALIE, FEROS, UVES and UES.

Valenti \& Fischer (2005) also included 94 Ceti in their spectroscopic study of cool stars. Their results are in agreement with the results of Santos et al., meaning that this star shows an overmetallicity with respect to the Sun. They also gave estimates of the age, mass, and radius of the star. Maldonado et al. (2012) spectroscopically analysed 94 Ceti as part of their survey of the metallicity of solar-like stars that have debris disks and planets. Their results are also in agreement with the previous mentioned analyses. A summary of the spectroscopic studies previously mentioned is presented in Table 1 .

\subsection{Interferometric observations}

Direct measurements for the radius of 94 Ceti A were performed with interferometric techniques by van Belle \& von Braun (2009). Using the Palomar Testbed Interferometer (PTI) and the CHARA array, they measured linear radii and effective temperatures of twelve stars with known planetary companions. For 94 Ceti A, a radius of $R / R_{\odot}=1.898 \pm 0.070$ was obtained, which provides an important constraint for stellar modelling. The associated effective temperature derived was $T_{\text {eff }}=6109 \pm 111 \mathrm{~K}$.

\subsection{The exoplanet data}

A Jupiter-like companion to 94 Ceti A was first announced by Queloz et al. (2000). A more precise orbital solution was later provided by Mayor et al. (2004). Discovered by using the radial velocity technique, this planet has a minimum mass of $1.68 M_{\mathrm{J}}$ and orbits its parent star at a distance of $1.42 \mathrm{AU}$ with a period of approximately 535.7 days. A summary of the properties of HD $19994 b$ is given in Table 2.
Table 3. Identified $P$-mode frequencies (in $\mu \mathrm{Hz}$ ) for 94 Ceti.

\begin{tabular}{cccc}
\hline \hline$l=0$ & $l=1$ & $l=2$ & $l=3$ \\
\hline 1224 & 1253 & 1218 & 1246 \\
1288 & 1316 & 1283 & - \\
1352 & 1381 & 1347 & - \\
1415 & 1445 & 1411 & 1438 \\
1479 & 1508 & 1475 & 1501 \\
1545 & 1572 & 1538 & - \\
1608 & 1637 & - & 1680 \\
1673 & 1702 & - & - \\
\hline
\end{tabular}

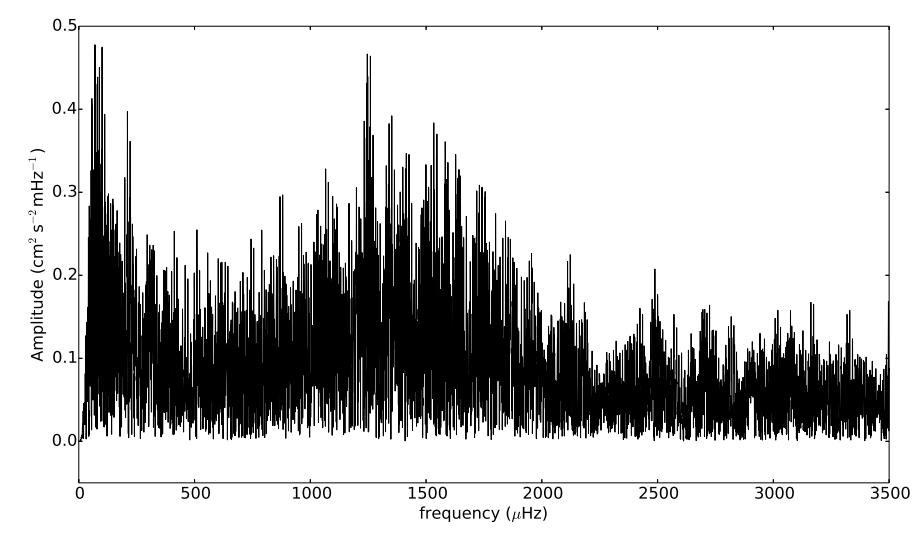

Fig. 1. Global Fourier transform of 94 Ceti data.

\section{Seismic observations and analysis}

\subsection{Mode identification}

We observed the star 94 Ceti A with the HARPS spectrograph mounted on the ESO $3.6 \mathrm{~m}$ telescope, at La Silla Observatory, Chile. We carried out the observations during seven consecutive nights in November 2007. The global Fourier transform of the observations is given in Fig. 1.

The Fourier transform was first analysed with the Period 04 code (Lenz \& Breger 2005) to extract the most important peaks. However, due to aliasing it may happen that high amplitude peaks are combinations of aliases instead of real modes. For this reason we also manually investigated the Fourier transform, using the window function to pick out the real peaks and eliminate the surrounding aliases. We then used stellar models to identify the modes corresponding to the detected frequencies. This method was somewhat successful, allowing us to recognise twenty-six modes in a convincing way. The frequencies of these identified modes are given in Table 3 with an uncertainty evaluated as $1 \mu \mathrm{Hz}$. The average large separation is $\Delta v=64.1 \mu \mathrm{Hz}$ and the average small separation is $\delta v_{0,2}=4.0 \mu \mathrm{Hz}$.

\subsection{Rotational splitting}

Given the projected rotational velocity $v \sin i$, the rotation period $P_{\text {rot }}$, and the linear radius $R_{\text {star }}$, the inclination angle $i$ of the rotation axis on the line of sight can be determined. In the case of 94 Ceti, $P_{\text {rot }}=12.2$ days (Mayor et al. (2004), from $R_{\mathrm{HK}}$ activity indicator) and $R / R_{\odot}=1.898 \pm 0.070$ from interferometric observations (van Belle \& von Braun 2009). We obtain sin $i \sim 1.02$ which means that the star is seen equator-on. Gizon \& Solanki (2003) showed that in this case, for $l=1$ modes, the central $m=0$ component should vanish. With a rotation period of $P_{\text {rot }}=12.2$ days, the shift induced by rotational splitting is 
$\sim 0.95 \mu \mathrm{Hz}$. Thus, in the 94 Ceti A case, we expect to see the $m= \pm 1$ components of $l=1$ modes separated by $\sim 1.9 \mu \mathrm{Hz}$. For $l=2$ modes, Gizon \& Solanki (2003) predict the visibility of the three components, such that $m=0$ and $m= \pm 2$. Also, the frequency shift between the visible components should be $\sim 1.9 \mu \mathrm{Hz}$. However, because the predicted amplitudes for the $m=0$ components are lower than those of the $m= \pm 2$ components, there may be cases where only $m= \pm 2$ components are visible. The present observations are not precise enough to test these rotational effects, because the uncertainties on the frequency determinations are of order $1 \mu \mathrm{Hz}$. However, this may be the reason for some of the shifts obtained between the observed and computed frequencies as seen in the échelle diagram (Fig. 3).

Because the star is seen almost equator-on, the mass of the planet HD 19994b should be close to its derived minimum value of $1.68 M_{\mathrm{J}}$. This planet may also be transiting in front of the star. To our knowledge, this sort of transit has not yet been looked for observationally.

\section{Asteroseismic study}

\subsection{Stellar models}

We used the Toulouse-Geneva Evolution Code (TGEC) to compute stellar models that fitted the seismic observations of 94 Ceti A. This code performs complete computations of atomic diffusion for twenty-one species, including radiative accelerations for all heavy elements. The twenty-one species include twelve elements and their main isotopes: $\mathrm{H},{ }^{3} \mathrm{He},{ }^{4} \mathrm{He},{ }^{6} \mathrm{Li}$, ${ }^{7} \mathrm{Li},{ }^{9} \mathrm{Be},{ }^{10} \mathrm{~B},{ }^{12} \mathrm{C},{ }^{13} \mathrm{C},{ }^{14} \mathrm{~N},{ }^{15} \mathrm{~N},{ }^{16} \mathrm{O},{ }^{17} \mathrm{O},{ }^{18} \mathrm{O},{ }^{20} \mathrm{Ne},{ }^{22} \mathrm{Ne}$, ${ }^{24} \mathrm{Mg},{ }^{25} \mathrm{Mg},{ }^{26} \mathrm{Mg},{ }^{40} \mathrm{Ca}$, and ${ }^{56} \mathrm{Fe}$ (Théado et al. 2012). The diffusion coefficients used in the code are those derived by Paquette et al. (1986). The Rosseland opacities are recalculated inside the model, at each time step and at every mesh point, using OPCD v3.3 and data from Seaton (2005), to take into account the local chemical composition. In this way, the stellar structure is consistently computed all along the evolutionary tracks, as well as the individual radiative accelerations of $\mathrm{C}, \mathrm{N}, \mathrm{O}, \mathrm{Ne}, \mathrm{Mg}, \mathrm{Ca}$, and $\mathrm{Fe}$. This is performed by using the improved semi-analytical prescription proposed by Alecian \& Leblanc (2004). A more detailed discussion of these computations is provided in Deal et al. (2016).

The equation of state used in the code is the OPAL2001 equation (Rogers \& Nayfonov 2002). The nuclear reaction rates are from the NACRE compilation (Angulo 1999). The mixing length formalism is used for the convective zones with a mixing length parameter of 1.8, calibrated on the Sun (Théado et al. 2009).

\subsection{Determination of the best model}

The stellar oscillation modes were derived for each model using the PULSE code (Brassard \& Charpinet 2008). We first computed models including diffusion, without the effects of radiative accelerations, with masses ranging from 1.36 to $1.50 \mathrm{M}_{\odot}$. The initial helium mass fraction $Y_{\text {init }}$ was chosen either as "solar" $\left(Y_{\text {init }}=0.271\right.$, Grevesse \& Noels 1993) or "galactic" $\left(Y_{\text {init }}=\right.$ $\left.Y_{\mathrm{G}}\right)$, the latter of which means that it increases with $Z$ following the chemical evolution of galaxies, as derived by Izotov \& Thuan 2004, 2010. The initial heavy-element mass-fraction $Z_{\text {init }}$ was chosen so that the surface metallicity at the present age lies inside the error bars of the observed values. This lead to $Z_{\text {init }}=$ 0.0245 for the low metallicity case $([\mathrm{Fe} / \mathrm{H}]=0.09 \pm 0.10)$ and to $Z_{\text {init }}=0.0305$ for the high one $([\mathrm{Fe} / \mathrm{H}]=0.21 \pm 0.08)$. The larger
Table 4. Properties of 94 Ceti from this work.

\begin{tabular}{cc}
\hline \hline & 94 Ceti \\
\hline$T_{\text {eff }}(\mathrm{K})$ & 6140 \\
$\log g$ & 4.041 \\
Mass $\left(M_{\odot}\right)$ & 1.44 \\
Radius $\left(R_{\odot}\right)$ & 1.89 \\
Luminosity $\left(L_{\odot}\right)$ & 0.66 \\
Age $(\mathrm{Gyr})$ & 2.38 \\
$Z_{\text {init }}$ & 0.0305 \\
$Y_{\text {init }}$ & 0.297 \\
$Z_{\text {surf }}{ }^{a}$ & 0.0268 \\
$Y_{\text {surf }}{ }^{a}$ & 0.243 \\
{$[\mathrm{Fe} / \mathrm{H}]^{a}$} & 0.17 \\
\hline
\end{tabular}

Notes. ${ }^{(a)}$ Values at the age of best model.

metallicities derived by Santos et al. (2004) with CORALIE and FEROS were not considered. Evolutionary tracks computed for these four different initial compositions are presented in Fig. 2.

For each track, we determined the model with the same average large separation as the observed one and then we derived the best of all these models for each initial composition. We did this by fitting the échelle diagrams with the observed one, using $\chi^{2}$ minimisations. This selection gave four potential candidates for the best model, one for each initial chemical composition. The first one has a mass of $1.40 M_{\odot}$ with $Y_{\text {init }}=Y_{\mathrm{G}}$ and $Z_{\text {init }}=0.0245$ at $2.32 \mathrm{Gyr}$ (bottom right panel of Fig. 2, model 1), the second one has a mass of $1.44 M_{\odot}$ with $Y_{\text {init }}=Y_{\mathrm{G}}$ and $Z_{\text {init }}=0.0305$ at $2.38 \mathrm{Gyr}$ (bottom left panel of Fig. 2, model 2), the third one has a mass of $1.42 M_{\odot}$ with $Y_{\text {init }}=0.271$ and $Z_{\text {init }}=0.0245$ at 2.79 Gyr (upper right panel of Fig. 2, model 3) and the last one has a mass of $1.44 M_{\odot}$ with $Y_{\text {init }}=0.271$ and $Z_{\text {init }}=0.0305$ at $2.97 \mathrm{Gyr}$ (upper left panel of Fig. 2, model 4). Comparing the $\chi^{2}$ values, we find that models 1 and 2 best fitted the observational values.

An interesting result of this study is the constraint on $\log g$. For all initial compositions used for these model the $\log g$ value ranges between 4 and 4.1. This rules out the spectroscopic $\log g$ and $T_{\text {eff }}$ determinations of Santos et al. (2004) obtained with the instruments CORALIE and FEROS, and the work of Valenti \& Fischer (2005) and Maldonado et al. (2012). On the other hand, the results obtained by Santos et al. (2004) with UVES and UES are consistent with the seismic determination of $\log g$

Comparing the seismic results with the spectroscopic observations (Fig. 2), we find that the best model is model 2, with a mass of $1.44 M_{\odot}$, because model 1 lies outside all spectroscopic boxes (the $1.40 M_{\odot}$ model on the bottom right panel of Fig. 2). The échelle diagram corresponding to this model is presented in Fig. 3. Its parameters are given in Table 4.

\section{Impact of radiative accelerations and fingering convection on the model parameters}

The radiative accelerations are larger than the gravity for stars with masses larger than $1.3 M_{\odot}$ at solar metallicity (Michaud et al. 1976). The best model for 94 Ceti has a mass of $1.44 M_{\odot}$. Even though the initial metallicity is larger than solar, the effect of radiative accelerations may be important and 

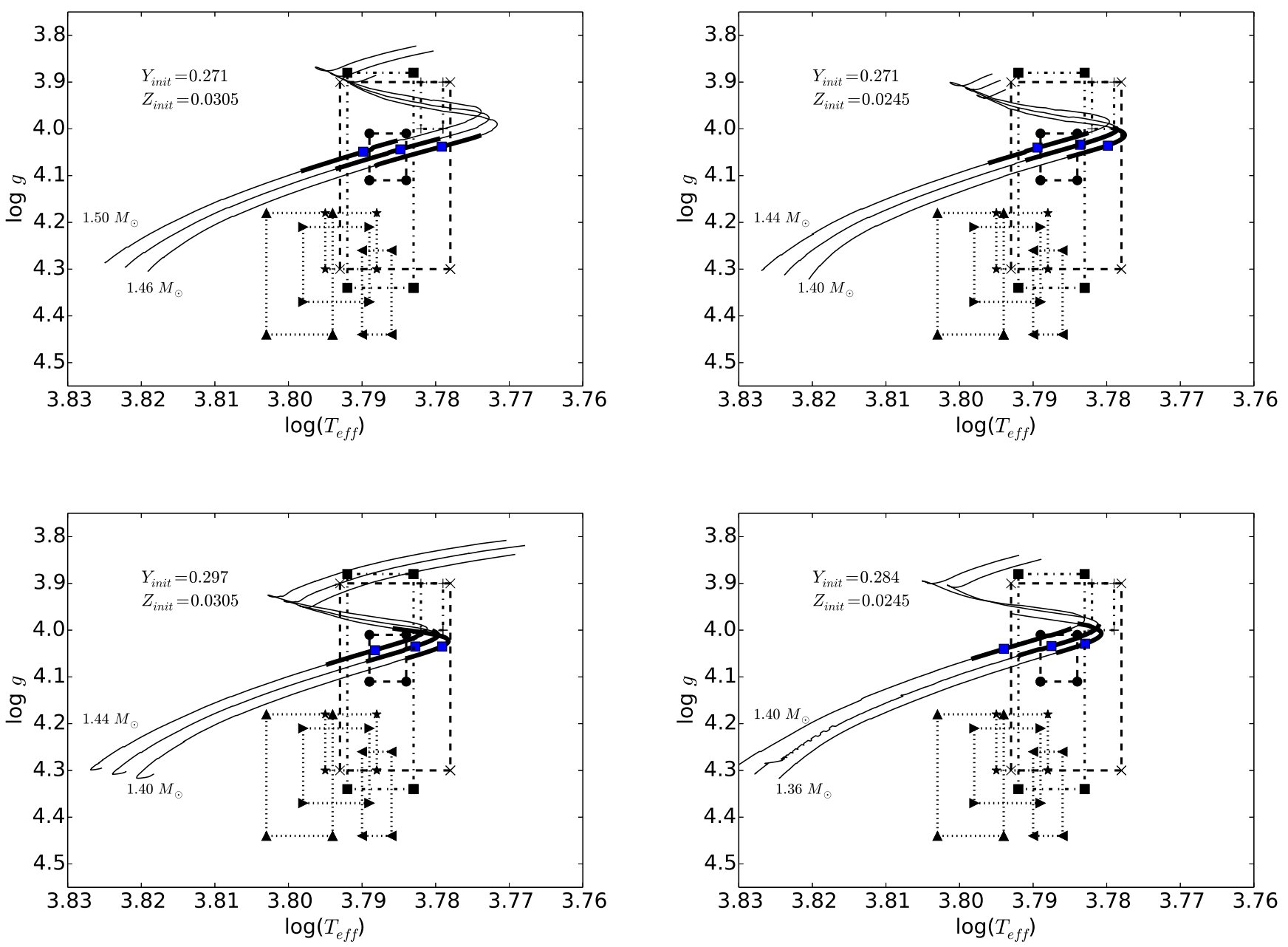

Fig. 2. $\log g$ vs. $T_{\text {eff }}$ diagrams for models with four different initial chemical compositions. The masses range between 1.36 and $1.5 M_{\odot}$. The error boxes come from the spectroscopic studies detailed in Sect. 2.1 and Table 1. The crosses correspond to Edvardsson et al. (1993), the pluses correspond to Smith et al. (2001), the right triangles correspond to Santos et al. (2004) (CORALIE), the triangles correspond to Santos et al. (2004) (FEROS), the dots correspond to Santos et al. (2004) (UVES), the squares correspond to Santos et al. (2004) (UES), the stars correspond to Valenti \& Fischer (2005) and the left triangles correspond to Maldonado et al. (2012). The blue squares represent the models with the same mean large separation as the one determined from the seismic observations. The heavy line segments correspond to models consistent with the interferometric radius.

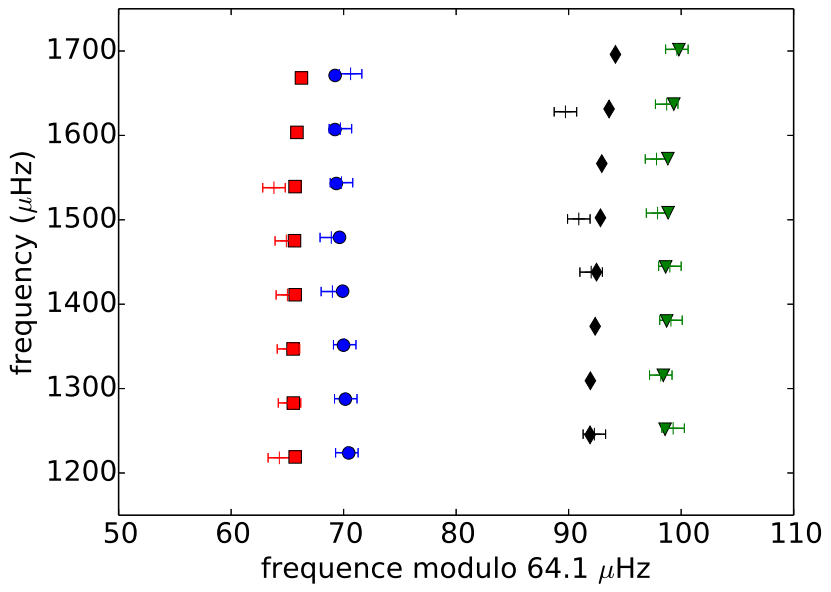

Fig. 3. Echelle diagram of the $1.44 M_{\odot}$ at $2.38 \mathrm{Gyr}$. The frequencies computed for the models with $Y_{\text {init }}=0.297$ and $Z_{\text {init }}=0.0305$ are represented by blue dots $(l=0)$, green triangles $(l=1)$, red squares $(l=2)$, and black diamonds $(l=3)$. The observed frequencies are represented by crosses (error bars: $1 \mu \mathrm{Hz}$ ). has to be investigated. As shown by Richer et al. (2000) and Richard et al. (2001), and more recently by Théado et al. (2009) and Deal et al. (2016), radiative accelerations that are larger than the gravity and decrease towards the surface may lead to element accumulation in specific stellar layers. These accumulations may also trigger fingering convection if they lead to inversions of $\mu$-gradients (Théado et al. 2009; Deal et al. 2016).

Our final aim is to evaluate the differences obtained for the best-model parameters when we include or not the radiative accelerations and the fingering convection in the computations. As a first step, we characterised the impact of radiative accelerations and fingering convection on the oscillation frequencies along evolutionary tracks. We then chose to compute the difference in ages of our best models, using the same initial conditions of mass and chemical composition.

\subsection{Impact of radiative accelerations}

We studied the impact of including radiative accelerations in the stellar models all along the evolutionary tracks upon the age determinations of the star. We used as initial conditions those of 


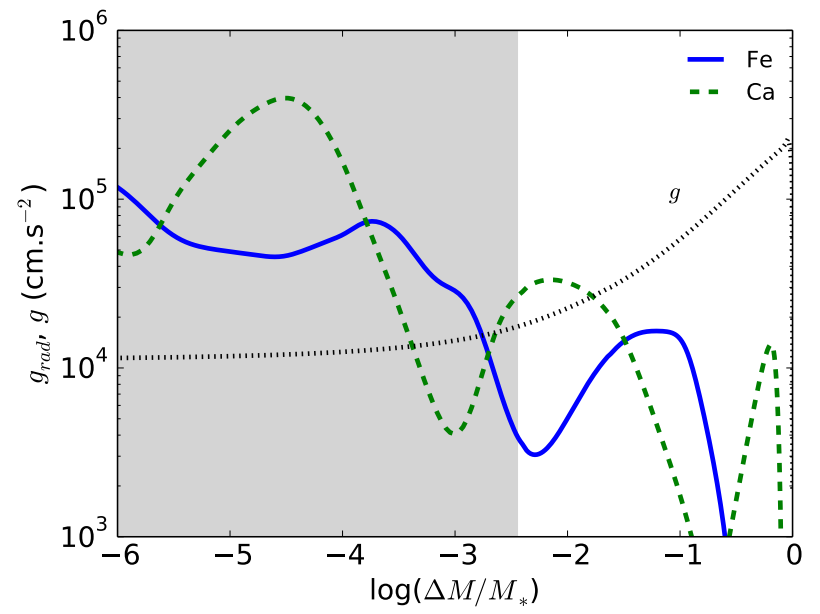

Fig. 4. Radiative acceleration on calcium (green dashed lines) and iron (blue solid lines) for the model of $1.44 M_{\odot}$ at $2.48 \mathrm{Gyr}$ with $Y_{\text {init }}=0.297$ and $Z_{\text {init }}=0.0305$. The dark grey area represents the surface convective zone.

the best model derived in Sect. 4.2. The mean large frequency separations computed in the models were used as a proxy.

We first computed a $1.44 M_{\odot}$ evolutionary track, including radiative accelerations all over the main sequence. The best model that we obtained in this case has an age of $2.48 \mathrm{Gyr}$. This is roughly $4 \%$ larger than the age determined without including radiative accelerations. The most important elements in this case are iron and calcium. As we see in Fig. 4, the radiative acceleration on calcium is larger than the gravity at the bottom of the surface convective zone. This produces an accumulation of calcium and increases the surface abundance (lower panel of Fig. 5).

Furthermore, although the radiative acceleration on iron is lower than the gravity in the radiative zone (Fig. 4), it is large enough to produce a gradient in the diffusion velocity and to slow down the gravitational settling of iron between $-2<$ $\log \left(\Delta M / M_{*}\right)<-1$. This leads to an iron accumulation at the bottom of the surface convective zone (lower panel of Fig. 5).

The abundance profiles are very different when radiative accelerations are taken into account. This modifies the mass of the surface convective zone from $\log \left(\Delta M / M_{*}\right)=-2.56$ when taking into account only gravitational settling to $\log \left(\Delta M / M_{*}\right)=-2.47$ when including radiative accelerations. This change of the mass of the convective zone by roughly $20 \%$ induces modifications of the oscillation frequencies that partly explains the difference in age obtained between the two models. Another reason for the difference in age is the modification of the opacity profile. Figure 6 shows the opacity profiles of the two models. The modification of the abundance profiles changes the opacity in the surface convective zone and at its bottom. This is due to the accumulation of calcium in the surface convective zone and the accumulation of iron around $\log \left(\Delta M / M_{*}\right) \sim-2$. These differences in the opacities are only of a few percent, but still have an impact on the mean density of the model and modify the age of the model for which the mean large frequency separation is consistent with the observations.

\subsection{Induced fingering convection}

Théado et al. (2009) and Deal et al. (2016) show that the accumulation of heavy elements in A-type stars due to the effect of radiative accelerations may produce an inverse mean molecularweight gradient and trigger fingering (thermohaline) convection.
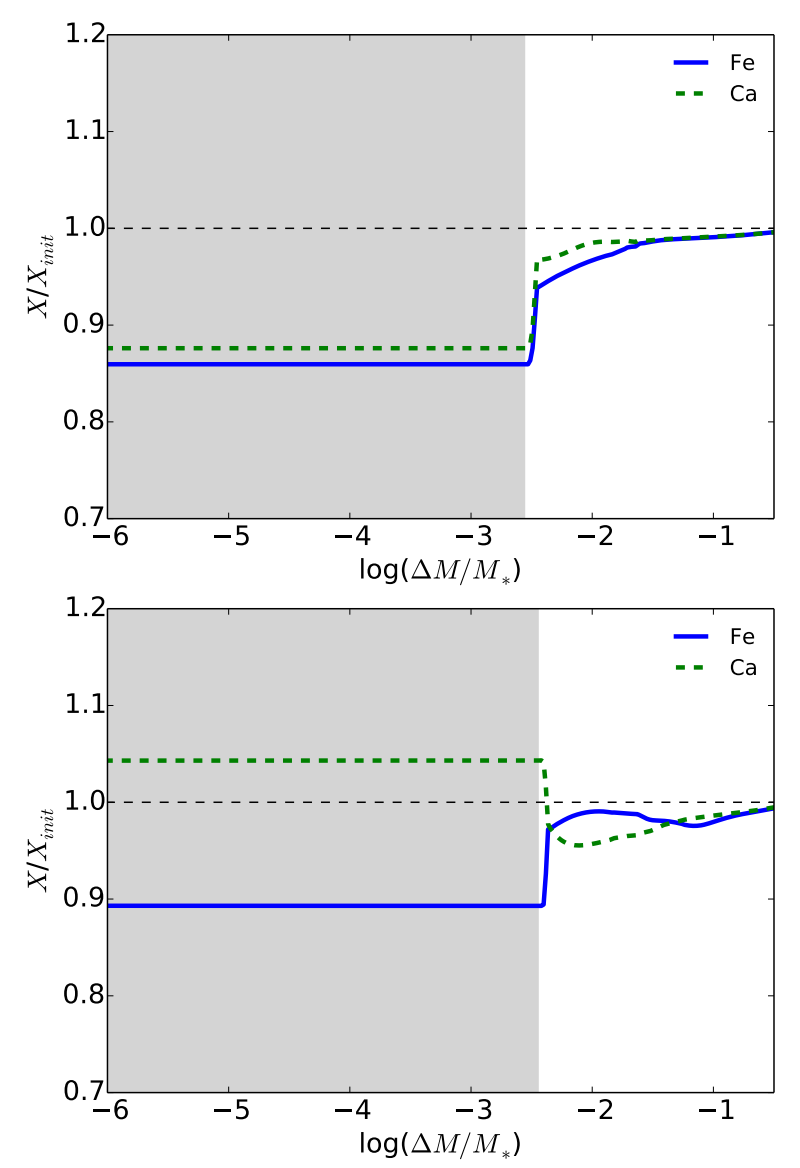

Fig. 5. Abundance profiles of calcium (green dashed lines) and iron (blue solid lines) for the model of $1.44 M_{\odot}$ with $Y_{\text {init }}=0.297$ and $Z_{\text {init }}=0.0305$ with gravitational settling only (upper panel) and including radiative acceleration (lower panel). The dark grey areas represent the surface convective zone.

This instability deeply modifies the structure of the star and should have an impact on the oscillation frequencies. In this section we study the impact of the fingering convection on the age determination for 94 Ceti A in the same way as in Sect. 5.1. Fingering convection may occur in stars every time a local inversion of the mean molecular-weight gradient appears in the presence of a stable temperature gradient. For example, this may happen in the case of heavy element accumulation or in the case of accretion of metal rich planetary matter (Vauclair 2004; Garaud 2011; Deal et al. 2013, 2015).

Fingering convection is characterised by the so-called density ratio $R_{0}$, which is the ratio between the thermal and $\mu$-gradients:

$R_{0}=\frac{\nabla-\nabla_{\mathrm{ad}}}{\nabla_{\mu}}$.

The instability can only develop if this ratio is higher than one and lower than the Lewis number, which is the ratio of the thermal to the molecular diffusivities. In this case, a heavy blob of fluid falls down inside the star and continues to fall because it exchanges heat with the surroundings more quickly than it exchanges particles. If $R_{0}$ is smaller than one the region is dynamically convective (Ledoux criterium), and if it is larger than the Lewis number the region is stable.

Various analytical treatments of fingering convection in stars were undertaken in the past, leading to mixing coefficients that 


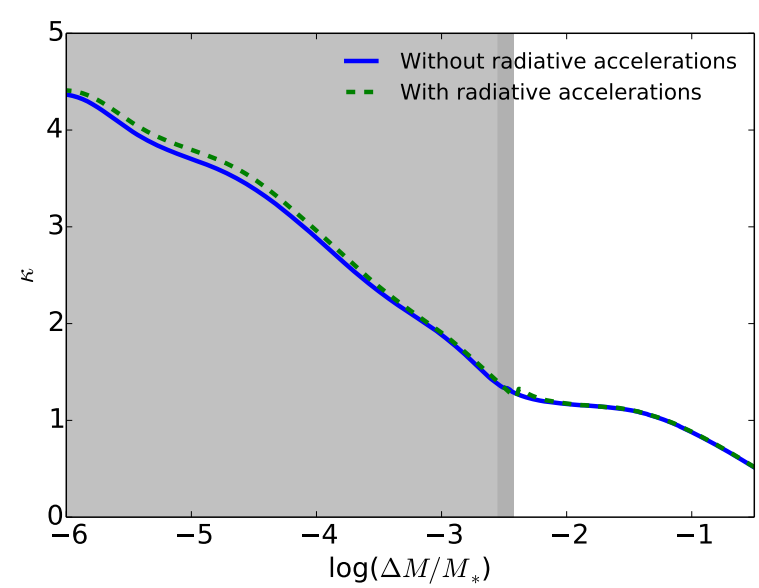

Fig. 6. Opacity profiles for the model of $1.44 M_{\odot}$ with $Y_{\text {init }}=0.297$ and $Z_{\text {init }}=0.0305$ with gravitational settling only (blue solid curve) and including radiative acceleration (green dashed curve). The light grey area represents the surface convective zone of the model when radiative accelerations are not included. The dark grey area represents the extension of this convective zone obtained when radiative accelerations are included in the computations.

differed by orders of magnitude (Ulrich 1972; Kippenhahn et al. 1980). More recently, 2D and 3D numerical simulations were performed that converged on coefficients of similar orders (Denissenkov 2010; Traxler et al. 2011).

Here we test the occurrence of fingering convection by using the recent prescription given by Brown et al. (2013) that has been confirmed by the 3D simulations of Zemskova et al. (2014). Whereas fingering convection may occur later on the evolutionary track, we found that in our best model for 94 Ceti the radiative layers are not yet subject to this instability.

The F-type star 94 Ceti lies in the range of effective temperatures for which radiative accelerations on individual elements begin to have important effects on the stellar internal structure. In a general way, the effects are larger for larger effective temperatures and lower metallicities. Many stars observed in the future by space missions such as TESS or PLATO will be affected by these sorts of effects. The characterisation of exoplanets needs very precise stellar parameters such as the mass, the radii and the age. With the precision of these new instruments, it will be necessary to always perform complete computations of atomic diffusion, including radiative accelerations as well as the induced mixing processes, for comparison with the observations.

\section{Effect of modifying the atmospheric boundary conditions}

When computing acoustic frequencies in stellar models obtained with the usual evolution codes, the waves are assumed completely reflected at some layer in the atmosphere. This is a crude approximation that becomes worse and worse for larger frequencies close to the cut-off value. This has been widely discussed in the literature, and a recipe has been provided by Kjeldsen et al. (2008) to try correcting the effect.

For the present paper, we chose to compute one complete model including a realistic atmosphere and compare the results with those of the same model computed with the usual atmospheric approximations. This is not trivial; we discuss below how we proceeded.

In stellar interiors, the medium is optically thick and the radiative transfer is computed using the diffusion approximation.
The outermost layers of a star being an optically thin medium, their structure requires the detailed computation of the radiative transfer. The approach we chose was to merge the internal structure of a TGEC model with a detailed ATLAS9 (Kurucz 1993) model atmosphere, to show the difference in the seismic properties obtained.

\subsection{Computation of the complete model}

In this section, we present the method used to merge a stellar interior with a realistic stellar atmosphere (Hui-Bon-Hoa 2013 contains further details). For a given TGEC model, we computed a model atmosphere with the same surface chemical composition and fundamental parameters. We merged the atmosphere and the interior at the layer where the temperature at the bottom of the atmosphere matches that of the interior. Once the atmosphere was anchored to the internal structure, slight adjustments were made to ensure continuity for the pressure. The density was then recomputed accordingly, assuming a perfect gas law. In the overlapping regions, the transition for each physical quantity from the interior to the atmospheric value was computed by using a third degree polynomial weighting.

The mixing length parameter $\alpha_{\mathrm{MLT}}$ is usually slightly larger in the internal structure than in the atmosphere, where observational constraints can lead to values as low as 0.5 (van't Veer-Menneret \& Megessier 1996). In our case, we checked that modifying the atmospheric mixing length parameter led to negligible effects on the computed frequencies.

\subsection{Effect on the mean large frequency separation}

We built a complete stellar model as described in Sect. 6.1, using the best TGEC model found in Sect. 5.2. This model has a mass of $1.44 M_{\odot}$, an initial composition $Y_{\text {init }}=0.297$ and $Z_{\text {init }}=0.0305$, an effective temperature $T_{\text {eff }}=6142 \mathrm{~K}$, and a gravity $\log g=4.0$. The average large separation found for the model without complete atmosphere is $64.1 \mu \mathrm{Hz}$. Computing the frequencies and separations in the new model including the complete atmosphere leads to a slightly smaller average large separation of $63.8 \mu \mathrm{Hz}$. This difference, smaller than $1 \mu \mathrm{Hz}$, is mainly due to the more realistic location of the outer turning point. Instead of being artificially set at the outermost layer of the internal structure model, it now occurs at the place where the atmosphere becomes radiative, that is to say where the Brunt-Väisälä frequency rises again. The échelle diagram obtained in this case is very similar to the one obtained from the original model. We thus conclude that our results described in the previous sections above are robust.

\section{Conclusion}

The observations of 94 Ceti A made with HARPS allowed precise parameters for this star to be obtained, resulting in the exclusion of several spectroscopic error boxes. Our best model lies inside the error boxes provided by Santos et al. (2004) that were obtained with UVES and UES observations. On the other hand, those obtained with FEROS and CORALIE give too large a gravity and too high an effective temperature.

We have tested the effect on the final results of including atomic diffusion with radiative accelerations, as well as induced fingering convection, in the models. The star 94 Ceti A is hot enough to have important radiative effects, in spite of its high metallicity reducing them. We found that the age of the final 
best model is a few percent larger than the age obtained without introducing radiative accelerations in the computations. This difference should be larger for hotter stars and for stars with lower metallicities. We note that this age difference may still be larger than the one derived in the present paper. We computed radiative accelerations by using SVP tables (Alecian \& Leblanc 2004) in which some elements are not yet included. Nickel, for example, may be important in that respect (Turcotte et al. 1998). Introducing a complete ATLAS atmosphere for the computation of the best model does not change the results in a significant way (less than one percent).

Considering the precision obtained with present and future space missions, our results show that complete atomic diffusion should be introduced in the models for stars with masses larger than 1.4 solar masses.

Acknowledgements. We thank the referee for his/her important remarks on the first version of this paper. The observations were performed with the HARPS spectrograph, ESO programme number 080.D-0408. We also thank the French Programme National de Physique Stellaire (PNPS) of INSU/CNRS for financial support. This work was performed using HPC resources from GENCICINES/IDRIS and from LUPM.

\section{References}

Alecian, G., \& Leblanc, F. 2004, in The A-Star Puzzle, eds. J. Zverko, J. Ziznovsky, S. J. Adelman, \& W. W. Weiss, IAU Symp., 224, 587

Angulo, C. 1999, AIP Conf. Ser., 495, 365

Brassard, P., \& Charpinet, S. 2008, Ap\&SS, 316, 107

Brown, J. M., Garaud, P., \& Stellmach, S. 2013, ApJ, 768, 34

Deal, M., Deheuvels, S., Vauclair, G., Vauclair, S., \& Wachlin, F. C. 2013, A\&A, 557, L12

Deal, M., Richard, O., \& Vauclair, S. 2015, A\&A, 584, A105

Deal, M., Richard, O., \& Vauclair, S. 2016, A\&A, 589, A140

Denissenkov, P. A. 2010, ApJ, 723, 563

Edvardsson, B., Andersen, J., Gustafsson, B., et al. 1993, A\&A, 275, 101

Garaud, P. 2011, ApJ, 728, L30
Gizon, L., \& Solanki, S. K. 2003, ApJ, 589, 1009

Grevesse, N., \& Noels, A. 1993, in Origin and Evolution of the Elements, eds. N. Prantzos, E. Vangioni-Flam, \& M. Casse, 15

Hale, A. 1994, AJ, 107, 306

Hui-Bon-Hoa, A. 2013, in EAS Publ. Ser. 63, eds. G. Alecian, Y. Lebreton, O. Richard, \& G. Vauclair, 35

Izotov, Y. I., \& Thuan, T. X. 2004, ApJ, 602, 200

Izotov, Y. I., \& Thuan, T. X. 2010, ApJ, 710, L67

Kippenhahn, R., Ruschenplatt, G., \& Thomas, H.-C. 1980, A\&A, 91, 175

Kjeldsen, H., Bedding, T. R., \& Christensen-Dalsgaard, J. 2008, ApJ, 683, L175

Kurucz, R. 1993, ATLAS9 Stellar Atmosphere Programs and $2 \mathrm{~km} \mathrm{~s}^{-1}$ grid. Kurucz CD-ROM 13 (Cambridge, Mass.: Smithsonian Astrophysical Observatory)

Lenz, P., \& Breger, M. 2005, Commun. Asteroseismol., 146, 53

Maldonado, J., Eiroa, C., Villaver, E., Montesinos, B., \& Mora, A. 2012, A\&A, 541, A 40

Mayor, M., Udry, S., Naef, D., et al. 2004, A\&A, 415, 391

Michaud, G., Charland, Y., Vauclair, S., \& Vauclair, G. 1976, ApJ, 210, 447

Paquette, C., Pelletier, C., Fontaine, G., \& Michaud, G. 1986, ApJS, 61, 177

Queloz, D., Mayor, M., Naef, D., et al. 2000, in From Extrasolar Planets to Cosmology: The VLT Opening Symp., eds. J. Bergeron, \& A. Renzini, 548

Richard, O., Michaud, G., \& Richer, J. 2001, ApJ, 558, 377

Richer, J., Michaud, G., \& Turcotte, S. 2000, ApJ, 529, 338

Rogers, F. J., \& Nayfonov, A. 2002, ApJ, 576, 1064

Santos, N. C., Israelian, G., \& Mayor, M. 2001, A\&A, 373, 1019

Santos, N. C., Israelian, G., Mayor, M., Rebolo, R., \& Udry, S. 2003, A\&A, 398, 363

Santos, N. C., Israelian, G., \& Mayor, M. 2004, A\&A, 415, 1153

Seaton, M. J. 2005, MNRAS, 362, L1

Smith, V. V., Cunha, K., \& Lazzaro, D. 2001, AJ, 121, 3207

Théado, S., Vauclair, S., Alecian, G., \& LeBlanc, F. 2009, ApJ, 704, 1262

Théado, S., Alecian, G., LeBlanc, F., \& Vauclair, S. 2012, A\&A, 546, A100

Traxler, A., Garaud, P., \& Stellmach, S. 2011, ApJ, 728, L29

Turcotte, S., Richer, J., Michaud, G., Iglesias, C. A., \& Rogers, F. J. 1998, ApJ, 504, 539

Ulrich, R. K. 1972, ApJ, 172, 165

Valenti, J. A., \& Fischer, D. A. 2005, ApJS, 159, 141

van Belle, G. T., \& von Braun, K. 2009, ApJ, 694, 1085

van Leeuwen, F. 2007, A\&A, 474, 653

van't Veer-Menneret, C., \& Megessier, C. 1996, A\&A, 309, 879

Vauclair, S. 2004, ApJ, 605, 874

Zemskova, V., Garaud, P., Deal, M., \& Vauclair, S. 2014, ApJ, 795, 118 